The theory that a toxin is the prime cause of Graves's disease, put forward respecticely by Jones of Bath and Thompson of New York, has nothing to support it in the cases quoted. Rest in bed and a milk diet are undoubtedly the correct treatment for all cases in the acute stage and if any good can be derived from the so-called intestinal antiseptics doubtless these cases will be benefited by them.

The percentage of cases with a history of acute rheumatisin in Dr. West's series is very closely borne out by my own series-21 per cent. in the former and nearly 19 per cent. in the latter : and it seems probable that in the future, with more care in obtaining thur history, many more cases will be found with a definite history of acute rheumatism than heretofore. It was not always asked for and in several instances it seems reasonably clear that what really was acute rheumatism has been loosely described as rheumatism or "a touch of rheumatism."

It appears, then, that though a definite relationship between Graves's disease and acute rheumatism has no been established yet the following questions may well be asked : 1. Is Graves's diseare due to a rheumatic diathesis ? 2 . Is it due to a toxin or toxins produced by acute rheumatism? 3. Is it an alternative set of symptoms to the more usual articular lesions in acute rheumatism? In cther words, is the hyperplasia of the thyroid gland a true rheumatic affection? And though one cannot at once hope for an answer yet it would seem to be a logical deduction from the above to treat a series of cases of Graves's disease in a routine way with sodium salicylate. The results would be highly interesting to compare with those already published.

Sonning, Berks.

\section{TIVO CASES OF OTITIC LATERAL SINUS PHLEBITIS, ONE COMPLICATED WITH CEREBELLAR ABSCESS. ${ }^{1}$}

BY DUNDAS GRANT, M.D., F.R.C.S.,

SLRGEON TO THE CEXTRAL LOXDOY THROAT AND EAR HOSPITAL.

CASE 1.-Thrombo-phlebitis of the bulb of the jugular vein and cerebellar alseess : ligature of the rein; later evacuation of the sinus and bulb; drainage of the cerebellar abscess; recovery. -The patient, a man, aged 28 years, was referred to me on Oct. 30th, 1905, on account of dulness of hearing and discharge. Previously he bad been giddy, with retching but not real vomiting; on the day before he had an attack of cold and shivering with actual vomiting. He had been the subject of chronic purulent discharge from the right ear of several years duration. His left foot was weaker than the right and the knee jerk was increased. His pupils were normal but he had slight nystagmus on looking to the left. The discharge was found to come from the attic and there was no perforation sound on inflation. There was no doubt as to the necessity for at once performing the radical mastoid operation and it seemed very probable that there was some more serious condition behind the disease in the middle ear.

On the following day the radical mastoid operation was performed at the Central London Throat and Ear Hospital in the usual way. The antrum was found full of granulation tissue, the disease extending into the mastoid cells which were opened up. The sickness continued for two days and when the vomiting passed off ineffectual retching still remained. The patient complained of great pain over his head and nystagmus was well marked when the eye was turned upwards, outwards, and to the left. The dressing was changed as required until Nov. 10th, when incontinence was noted and the temperature rose to $103 \cdot 6^{\circ} \mathrm{F}$. The blood count showed 4,800,000 red cells and 19,500 leucocytes per cubic millimetre, 60 per cent. being multinuclear and $40 \mathrm{lym}$ phocytes; there were no bacteria. On the 11th in the morning the patient had a rigor lasting 16 minutes. The temperature was $103 \cdot 8^{\circ}$ and in the afternoon he felt hot and had been sweating profusely for two hours.

The second operation was performed, the old wound being reopened and an incision carried backwards at right angles

1 A paper rear berore the Otological Society of Great Britain and cases shown on Feb. 5 th, 1906 . to it along the course of the right lateral sinus. The bone was caretully chiselled away over the course of this vessel which, when exposed, presented a healthy appearance in its posterior part but was abnormally white in the anteroinferior portions, the walls being apparently thickened. No fluctuation impulse could be obtained with one finger on the course of the internal jugular vein in the neck and the other on the vertical portion of the sinus. This was taken as evidence that there was thrombosis between these two points. The sinus was now slit open and found to be partially thrombosed. Blood flowed freely from it above and this was stopped by plugging; a smaller stream oozed from below and this also was temporarily plugged. I then exposed the internal jugular vein in the neck above the entrance of the common facial; it was so collapsed and small as almost to simulate the vagus nerve and the common facial was distended as if it had already begun to take up the collateral circulation. Ligatures were applied at two places above the entrance of the common facial, the lower being the first tied ; from the upper part before ligature blood was allowed to ooze. A probe was passed up into the bulb of the jugular and then a syringe was placed in the lumen of the vein to exercise suction but no pus or clot was withdrawn; the wound in the neck was stitched with silk sutures, a small tube being left in for drainage. Going back to the retroauricular operation wound, the plug from the lower part of the sinus was removed and the freest possible flow was allowed to continue for a short time for the purpose of washing out the channel. A plug was then re-inserted.

On Nov. 12th in the morning the patient was subjectively much better; the temperature was $99 \cdot 6^{\circ}$ and the pulse and respirations were in proportion. The head was clear and the tongue was moist. At 9.30 in the evening the patient bad a rigor and $I$ removed the plugs frc $m$ the post-aural cavity and wound in the lower part of the sinus. From the latter, venous. blood oozed to some extent and the plug was therefore reinserted. The drainage-tube from the wound in the neck was withdrawn; slight facial paresis, involving chiefly the fibres. going to the lips, being then noted. On the 13th an injection of seven cubic centimetres of antistreptococcic serum was given; two hours later the patient was drowsy and the cerebration was slow. The dressing was changed and : ome sloughing was found to be taking place in the depth of the wound in the neck, which was syringed with biniodide of mercury lotion. The post-aural wound was opened freely, c'eansed, and re-dressed. The temperature oscillated between $98 \cdot 4^{\circ}$ and $104 \cdot 8^{\circ}$. The serum was repeatedly injected, as marked on the chart. On the 14th the patient's temperature remained unaltered and his condition :eemed hopeless. At. 7.30 at night a subcutaneous transfusion of normal saline solution, to the extent of one pint, was carried out, the place selected being the tissues under the skin of the left thigh. (the process lasting 20 minutes). At 10 P.M., two hoursafter the injection of the saline fluid, the temperature fell and remained nearly normal. On the 15th the dressings were changed and there was no rigor. On the 16th the temperature rose to $99 \cdot 8^{\circ}$ in the evening: and when the dressing was changed much foetid pus. was observed in the wound behind the ear. On the 17 th the temperature was normal but there was still a large quantity of foetid pus. On the 18th the patient was dressed at 2 P.M. and two and a half hours later it was noted that he was becoming extremely drowsy, a slighter degree of the condition having been noted by the matron earlier in the day; he bad also complained of headache. The drowsiness was more conspicuous in the evening and was deepening into coma; it was only with difficulty that he could be got to give replies to questions. The pupils reacted still to the light and were $\epsilon$ qual. Nystagmus was present as before and it was now noted that the right hand was weake than the left, a phenomenon which had been previously sought for and found wanting (the patient is a right-handed man). On the 19 th, the same comatose condition being now more marked, lumbar puncture was performed and the cerebellum and temporo-sphenoidal lobe were explored. The lumbar puncture failed owing to the blunting of the point of the needle against the lamina of the vertebra, so that it did not enter the theca. The cerebellum was exposed behind the posterior border of the mastoid and below the inferior curved line of the occipital bone; it bulged distinctly and an exploratory incision was made by means of a long narrow $\mathrm{knife}$ in the direction of the internal ear, also in other directions, and an exploring cannula was inserted but no pus was found. The dura mater was stitched together, as were also. 
the skin and deeper structures. The temporo-sphenoidal lobe inserted my finger through the trephine opening and found was next explored and the meninges over it were found to that it entered a cavity of roughly about half an ounce in be bulging, but an exploratory incision and the insertion capacity, with smooth walls. Into this I introduced a of the cannula evacuated no pus. Hæmorrhage result- narrow piece of indiarubber tubing and two plugs of ing from the division of the middle meningeal was checked iodoform gauze, the extremities of these being brought out by pressure. The dura mater and soft parts were sutured; through the opening in the reflected section of the flap. The the wound behind the auricle was again investigated and anterior part of the cerebellum to the central site of the thoroughly cleansed. The temperature rose for one reading remains of the sinus was soft and ragged and it now seemed to $104^{\circ}$ and then descended to $96^{\circ} 8^{\circ}$, remaining, as will be clear that the bulk of the pus had come from a cerebellar seen, much below the normal. On the 20th in the depth of abscess discharging in this position. On Nov. 25th the plugs the wound there was a considerable amount of fœitid pus; were removed from the cerebellum and found to be fœetid; the parts were cleansed with chlorinated soda, a plug the plugs from the neck and post-auricular incision were moistened with this being left in situ. On the 21st the quite free from foetor, the abscess in the cerebellum appafotor was not so prominent and the wound in the neck rently relieving itself entirely through the posterior tube looked more healthy. On the 23rd the patient was still in which was withdrawn to the extent of about one inch. On a semi-comatose condition, dozing most of the day and the 26 th the wound in the neck was still inactive and answering questions only very slowly when roused. It was covered with brownish pus. There was also some fœetid again found that the grip of the right hand was less strong brownish pus again found in the antro-tympanic cavity than that of the left and that sometimes the patient was and welling up from the cerebellum. The whole wound seen to lift the right hand by means of the left one. There was carefully irrigated with boric acid and swabbed was some bogginess over the cerebellar incision and on with biniodide solution; a second very small tube was pressure fotid pus flowed out. The cavity was cleansed and inserted into the cerebellum and through it the abscess the parts were very lightly plugged, boric fomentations being l cavity was gently syringed with boric lotion, some pus

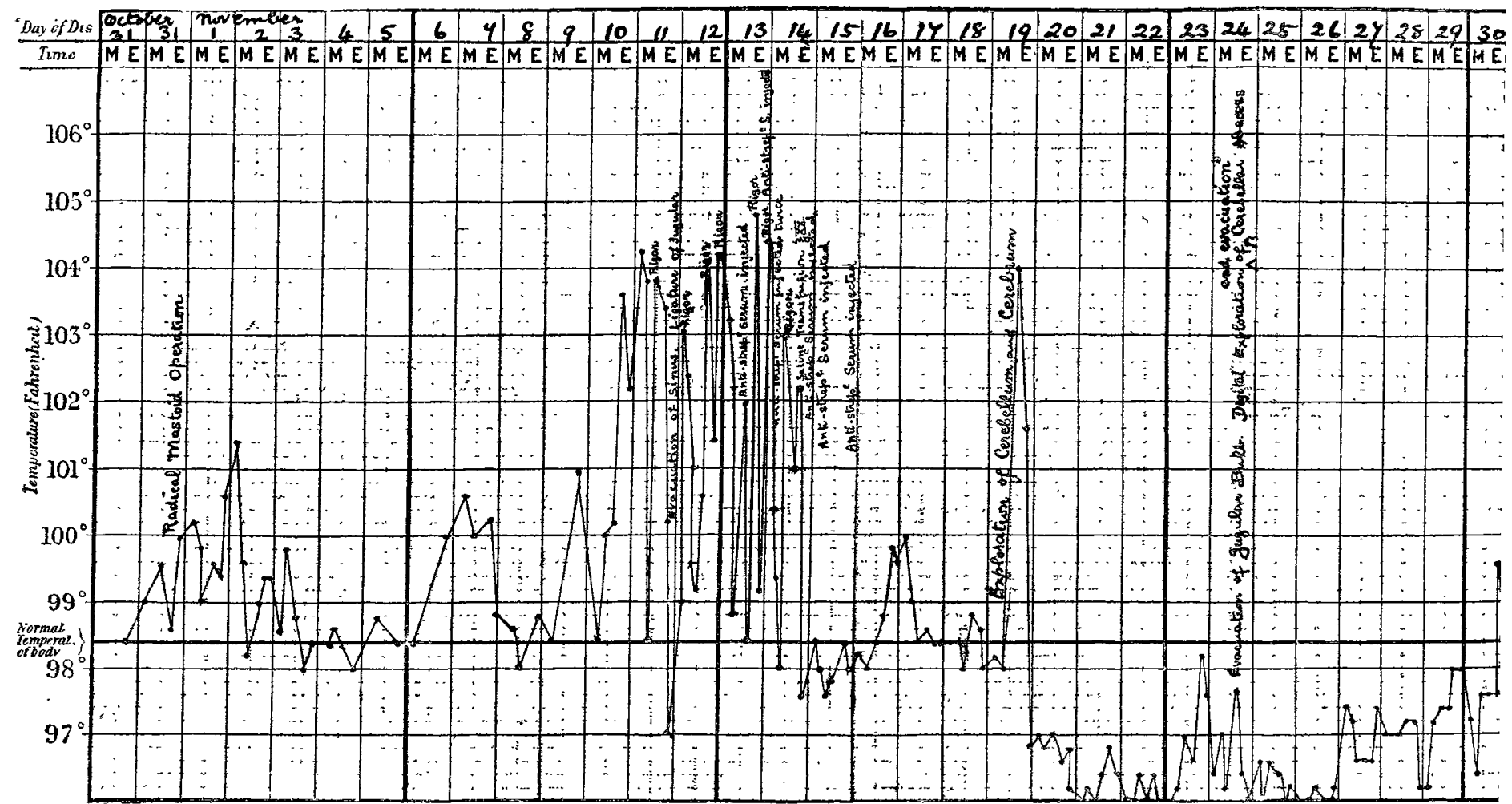

Pulse and respirations were practically proportional to the temperature throughout.

applied. On the 24th the state of the patient remained very much the same but it was noted that on pressure above, pus flowed along the course of the right internal jugular vein the pus (according to the notes) was thick and creamy, differing from that behind the auricle, which was fœtid, and that coming from the cerebellar incision, which was thin, brown, and fotid. At 6 o'clock in the evening, the condition remaining as above, a probe was inserted into the sinus at the base of the wound in the neck and it was found that it led upwards towards the angle between the mastoid process and the angle of the jaw; another probe was put in the course of the lateral sinus, behind the petrous portion of the mastoid, but the two did not touch.

In order to explore the bulb of the jugular vein I carried the post-auricular incision downwards to meet the wound in the neck and reflected the soft parts off the mastoid process, as also the periosteum, cutting through the posterior belly of the digastric after the superjacent muscular fibres. The mastoid process was chiselled away still further to expose the lateral sinus, and more especially the portion of bone dipping down between the sigmoid sinus and the bulb of the jugular vein, this part of the vessel being freely opened and cleansed. The source of the fœtid pus was, however, not so far discovered. The cerebellum was then once more exposed, but exploration with the knife and pusseeker afforded, as before, no signs of an abscess. I then being thus extruded; the second tube was left in situ; the wound was lightly packed and boric fomentations were applied and changed every six hours. (The pus was examined on the 27 th ; in it diplococci predominated, which retained the Gram stain; there were a few intracellular micrococci and a few chain cocci.) On the 27th, when the tube was taken out of the cerebellum, the pus, which welled up, was foetid and darker than on the previous day. The tube was re-inserted and the fomentations were re-applied. In the afternoon I withdrew from the cerebellar cavity, by means of suction, some pus and disintegrated cerebellar substance. The tube was re-inserted and fomentations were re-applied. On the 29th the brownish pus which exuded wben Dr. G. F. Buchan dressed the wound was in smaller quantity and the pulsation was very marked. The tube was reduced in size and the dressings and fomentations were re-applied. The patient on this day said that he felt much better; his intellect was considerably brighter and his replies to questions were more readily given. He sometimes spoke of his own accord and took more food; his pulse was more regular and of fair quality. On the 30th the wound loosed more healthy; the pus from the cerebellum, though still foetid, was smaller in quantity; the patient was hungry and continued to have a good appetite. The temperature was $99 \cdot 5^{\circ}$ but fell to normal and from this time forward was on or below the normal line. On Dec. 2nd his general condition was better; his pulse was stronger and of better quality. 
his mental condition was quite clear, the wound looked healthy, and the pus from the cerebellum was less fœetid.

From this time the improvement was steady and on Dec. 6th the tube was permanently removed from the cerebellum. The wound began to close rapidly; healthy granulations were present and it has steadily diminished ever since, the patient improving and being now free from all constitutional disturbance. On Jan. 11th, 1906, a small flat sequestrum from the edge of the cerebellum opening was removed and drying powder, devised by Mr. W. Stuart-Low, was dusted over the granulating surface (under this powder, with which Mr. Stuart-Low is making experiments, the epidermisation appeared to proceed with considerable rapidity). On the 18th a granulation was removed from the depth of the tympanum and spirit drops were ordered to be inserted.

The above case presents in a very pronounced way the symptoms characteristic in the first place of otitic pyæmia and subsequently of cerebral or cerebellar abscess. Among the features which seem most interesting are the following. 1. The positive evidence of thrombosis in the jugular bulb. 2. The negative results of exploration of the cerebellum through the post-mastoid trephine opening. In similar circumstances an exploration through the anterior part, on the central site of the sigmoid sinus, would probably have been more positive in results. 3. The effect of the subcutaneous injection of a quantity of normal saline solution which seemed to have been one of the turning points in the case, the antistreptococcic serum which was used, somewhat experimentally, being apparently devoid of result.

CASE 2. Lateral sinus thrombosis treated by operation without ligature of jugular vein; recovery. - The patient, a girl, aged 14 years, was first seen on Dec. 1st, 1904, complaining of headache, giddiness, and vomiting. She had been the subject of suppuration in the middle ear for two years and had been suffering from her present symptoms for a fortnight, having had several shivering attacks. Her temperature on admission was $105^{\circ} \mathrm{F}$. It was decided to operate at once and the radical mastoid operation was carried out, with the result that the cavities of the tympanum and antrum were found full of granulation tissue but quite free from purulent discharge of any kind. This suggested that the absence of discharge might be looked upon as an indication that there was a purulent focus elsewhere. An opening was therefore chiselled into the bone behind at a slightly higher level than the antral opening ; pus escaped under pressure forcing its way up at the side of a mass of granulation tissue. On enlarging the opening it was found that the pus was coming not merely from the groove of the sinus but from the interior of the venous channel itself, which was filled with granulation tissue and broken-down clot. The brokendown portion was scraped away till blood flowed freely from the posterior part of the sinus into which an iodoform gauze plug was then placed. At the lower part there was found apparently healthy organising clot and this was left in situ. The wound was lightly plugged with gauze and an antiseptic dressing was applied. Two days later all the plugs were removed; no hæmorrhage took place. The temperature became normal and never rose above $99 \cdot 4^{\circ}$. No flaps were cut in the meatus, as it was hoped that what the operator had previously seen would take place-namely, that the membranous part of the meatus would gradually dilate and become fixed to the walls of the cavity by contracting fibrous tissue. This did not take place at the time and indeed the passage was so narrow as to cause some disappointment. A little discharge also persisted from the deeper parts. During the next two months the discharge was frequently sucked out by means of Siegel's speculum and spirit drops were inserted at intervals of one or two days. Since then the meatus gradually dilated, so that a sufficient opening is left, and it is believed that as this process of centrifugal contraction goes on it will be quite as wide as could be desired. At the present moment there is no discharge and the meatus is quite large.

This case illustrates very typically the form of lateral sinus thrombosis in which it is justifiable to omit the ligature of the jugular vein and to limit the radical intervention to the temporal bone and sinus. A number of such cases are quoted by Nacewen in his work on the "Pyogenic Diseases of the Brain and Spinal Cord."

Cavendish-square, $\mathrm{W}$.

\section{THE TURK'S AND CAICOS ISLANDS AS A RESORT FOR CASES OF PULMONARY TUBERCULOSIS.}

By GEORGE S. S. HIRST, M.B., C.M. EDIN.

THE Turk's and Caicos Islands, though practically unknown to the majority of people, form a dependency of Jamaica and lie between $21^{\circ}$ and $22^{\circ} \mathrm{N}$. latitude and $71^{\circ}$ and $72^{\circ} 37^{\prime \prime} \mathrm{W}$. longitude. Geographically they belong to the Bahamas group of islands but since 1873 they have been constituted a dependency of Jamaica. Grand Turk, the capital of the dependency, is in direct communication with New York by the Clyde line of steamers, with Halifax by Messrs. Pickford and Black's boats, and with Bristol by the Elder-Dempster line.

Though thousands of people visit Jamaica from New York and Great Britain every year either in search of health or pleasure these islands have been entirely overlooked by the tourist and invalid. To the former they offer little or no attraction, but to the latter their claims are certainly worthy of consideration, for the climate is healthy and equable, the heat never being oppressive on account of the never-failing breeze day and night, and living is cheap.

The population of the islands is divided into white, coloured, and black. There are about 500 whites, of whom the majority have been born in the dependency and who consequently look upon the islands as their home. Of the remainder, some have come to the dependency suffering from pulmonary tuberculosis in the hopes of prolonging their lives and have lived 30 or 40 years in apparent good health.

The climate of the islands will be best judged from the following meteorological table:-

Meteorological Observations at the Settlement of Salt Cay of the Turk's Islands Group for 1905.

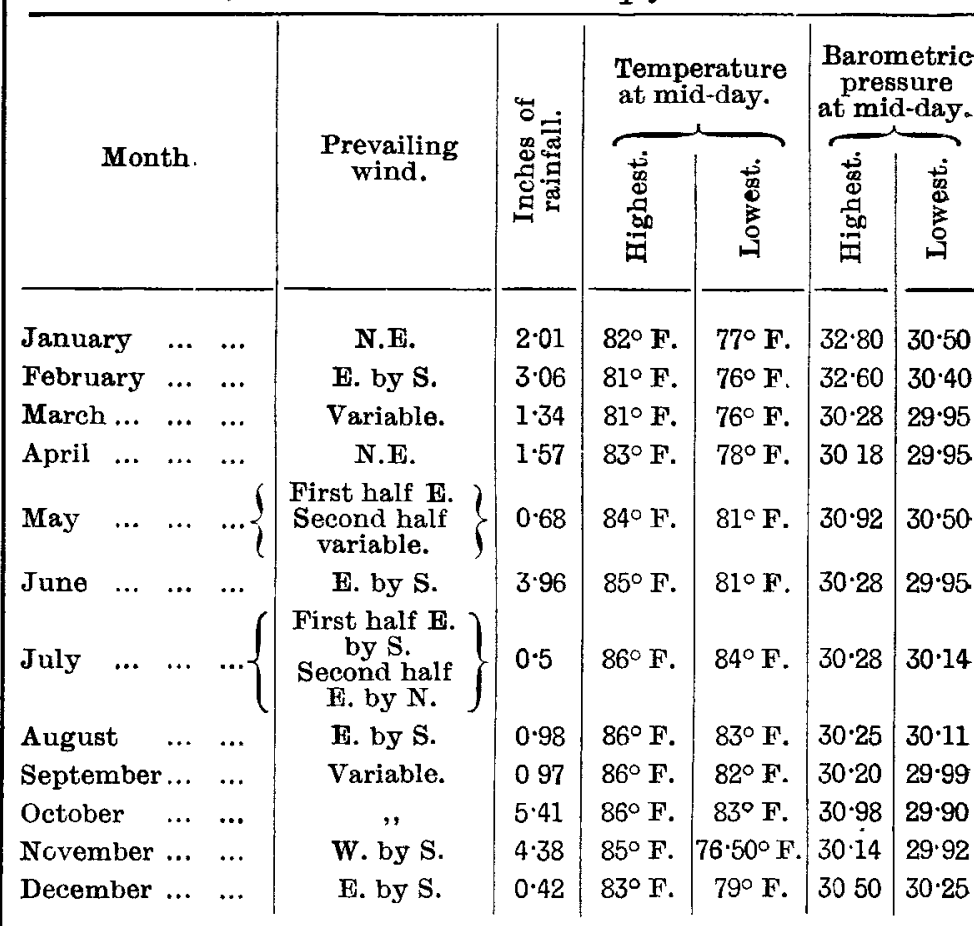

The above table applies only to the island of Salt Cay, but it would apply with slight variations to the other islands forming the group, except as regards the rainfall, and this differs within large limits, on some of the islands the rainfall recorded being double that at Salt Cay. Again, the rainfall differs on each island from year to year within quite large limits. The hours of sunshine have not been recorded because during the day the sun is practically always shining. A whole dull day occurs but rarely. In the months of August and September, known as the "hurricane months," the wind blows with greater force than at any other period of the year. Twice in the last century during the month of August-viz, in 1866 and 1887-a hurricane visited the islands, causing considerable damage to property. The 1899 hurricane, which did such damage in Jamaica, missed these islands. 